\title{
In Silico Antibody Mutagenesis for Optimizing its Binding to Spike Protein of Severe Acute Respiratory Syndrome Coronavirus 2
}

\author{
Binquan Luan* and Tien Huynh \\ Computational Biological Center, IBM Thomas J. Watson Research, Yorktown Heights, \\ New York 10598, USA \\ E-mail: bluan@us.ibm.com
}




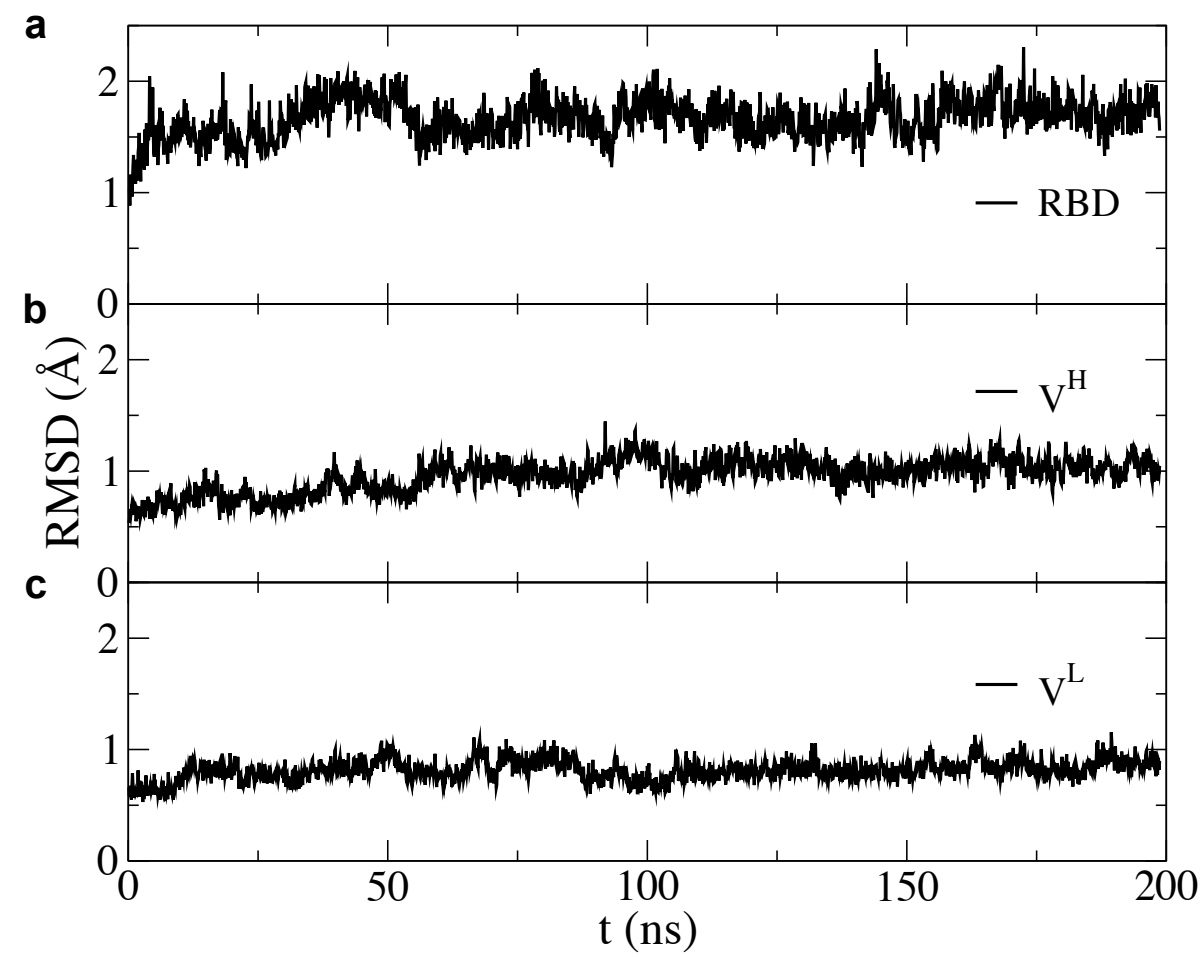

Figure S1: MD simulation of Fab-RBD complex. a) Root mean square deviations (RMSDs) of heavy atoms (i.e. nonhydrogen atoms) in RBD's backbone. b) RMSDs of heavy atoms in $\mathrm{V}^{H}$ 's backbone. c) RMSDs of heavy atoms in $\mathrm{V}^{L}$ 's backbone.
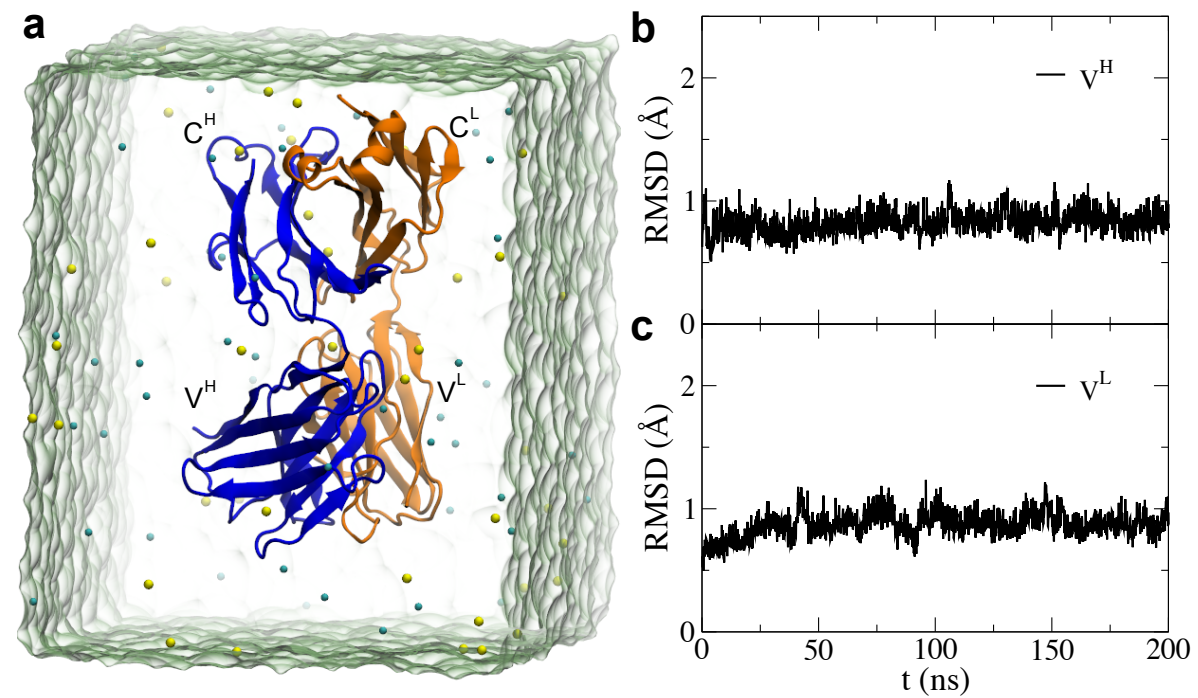

Figure S2: MD simulation of Fab only. a) Simulation system. The light chain in Fab is colored in orange and the heavy chain (partial) is colored in blue. Water is shown transparently; $\mathrm{Na}^{+}$and $\mathrm{Cl}^{-}$ions are shown as yellow and cyan spheres, respectively. b) RMSDs of heavy atoms in $\mathrm{V}^{H}$ 's backbone. c) RMSDs of heavy atoms in $\mathrm{V}^{L}$ 's backbone. 

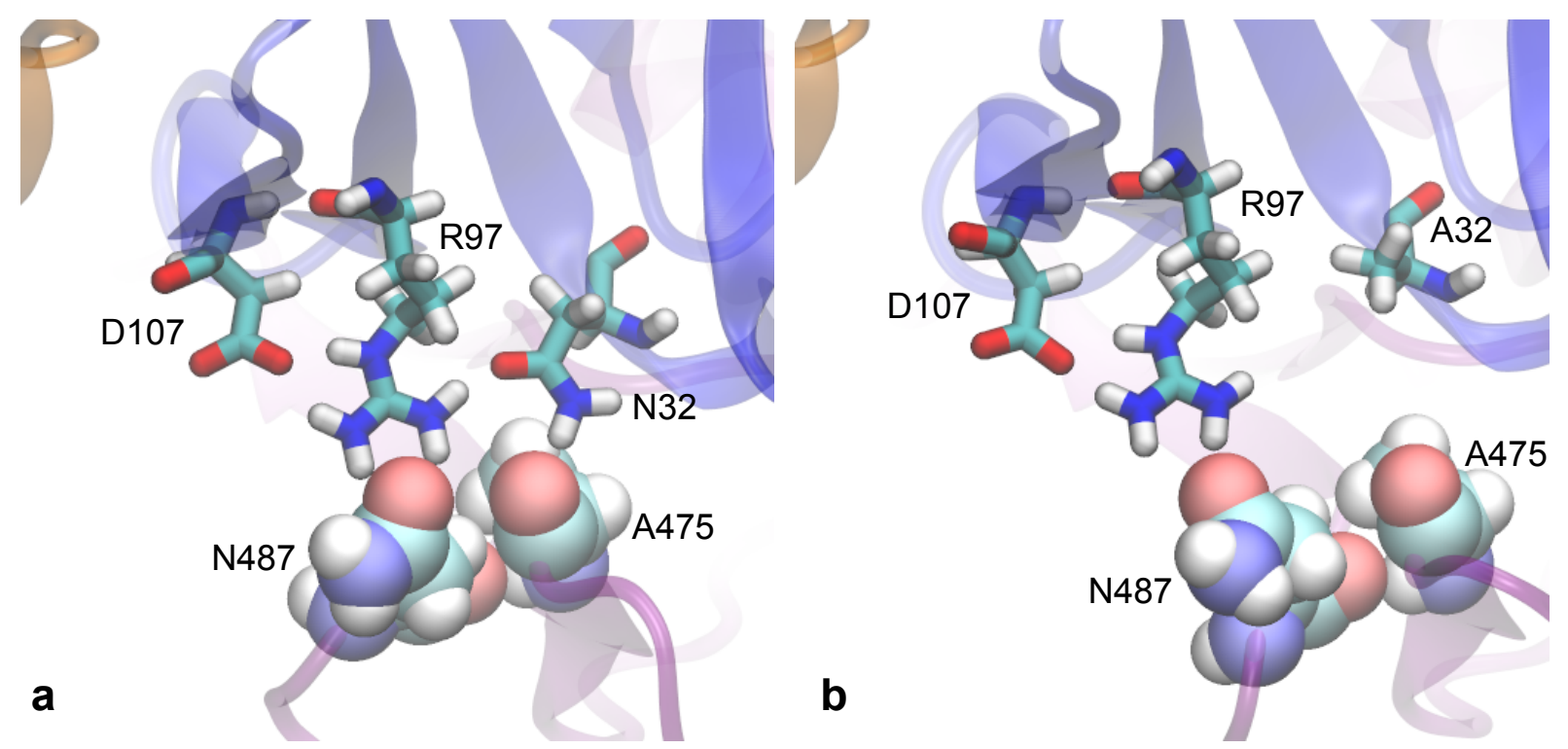

Figure S3: Illustration of the N32A mutation. a) Before the mutation. N32 formed a hydrogen bond with $\mathrm{A} 475$ in the RBD and another hydrogen bond with R97 in the $\mathrm{V}^{H}$. R97 interacted with N487 in the RBD strongly, when being sandwiched between D107 and N32. b) After the mutation. A32 was not in contact with any residue in the RBD. Without interaction with A32, R97 moved towards D107 and had a weaker interaction with N487 in the RBD. Overall, the local interfacial interaction was reduced after the N32A mutation. 


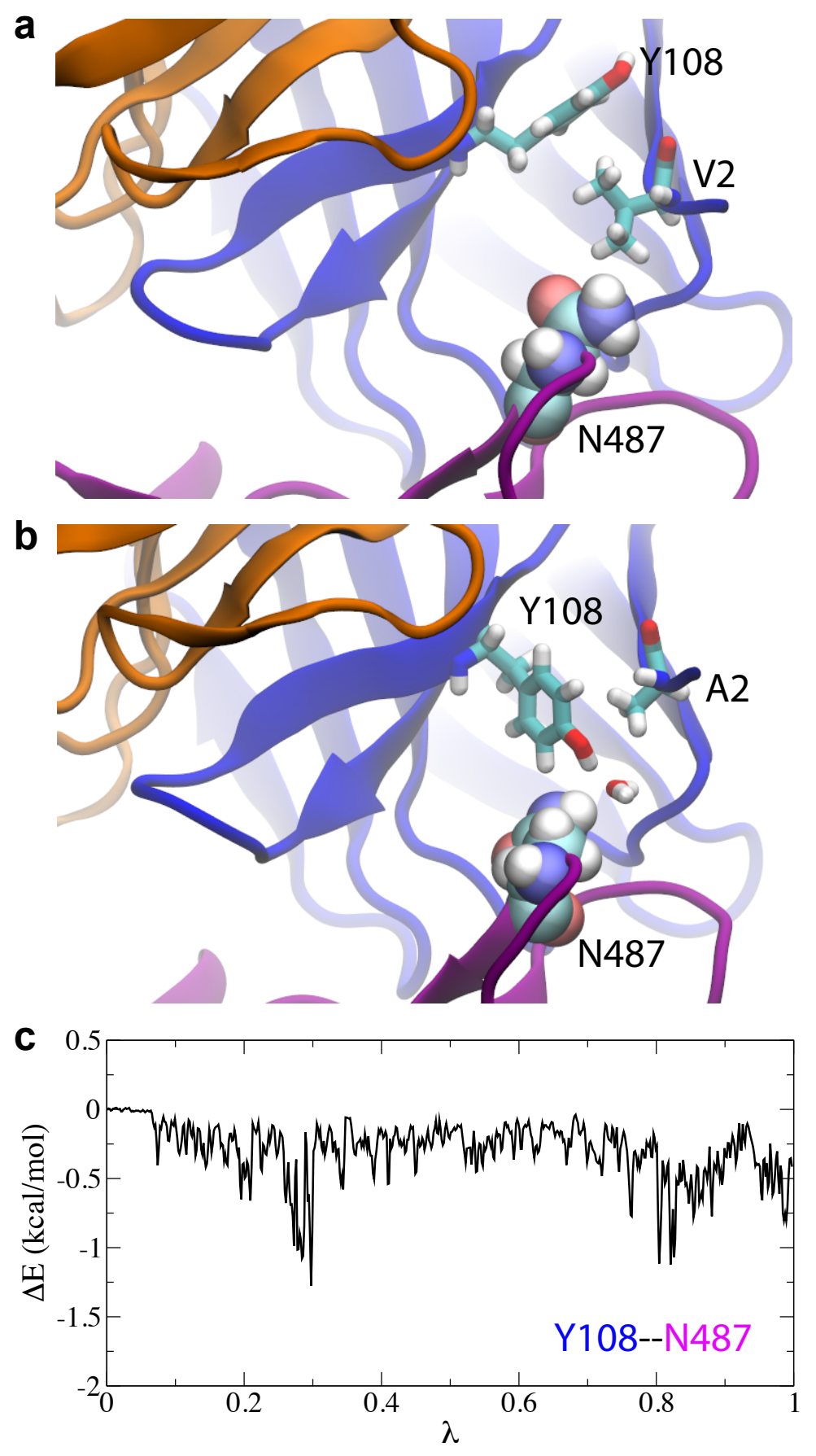

Figure S4: Illustration of the V2A mutation. a) Before the mutation. the bulky V2 prevented Y108 from moving to the interface. In the free state, Y108 resided at the similar location. b) After the mutation. Y108 arrived at the interface and interacted with N487 in the RBD. c) The interaction potential energy (mainly from the van der Waals one) between Y108 in the $\mathrm{V}^{H}$ and $\mathrm{N} 487$ in the RBD. In average, this interaction energy reduced about $0.5 \mathrm{kcal} / \mathrm{mol}$ during the $\mathrm{V} 2 \mathrm{~A}$ alchemy process. 


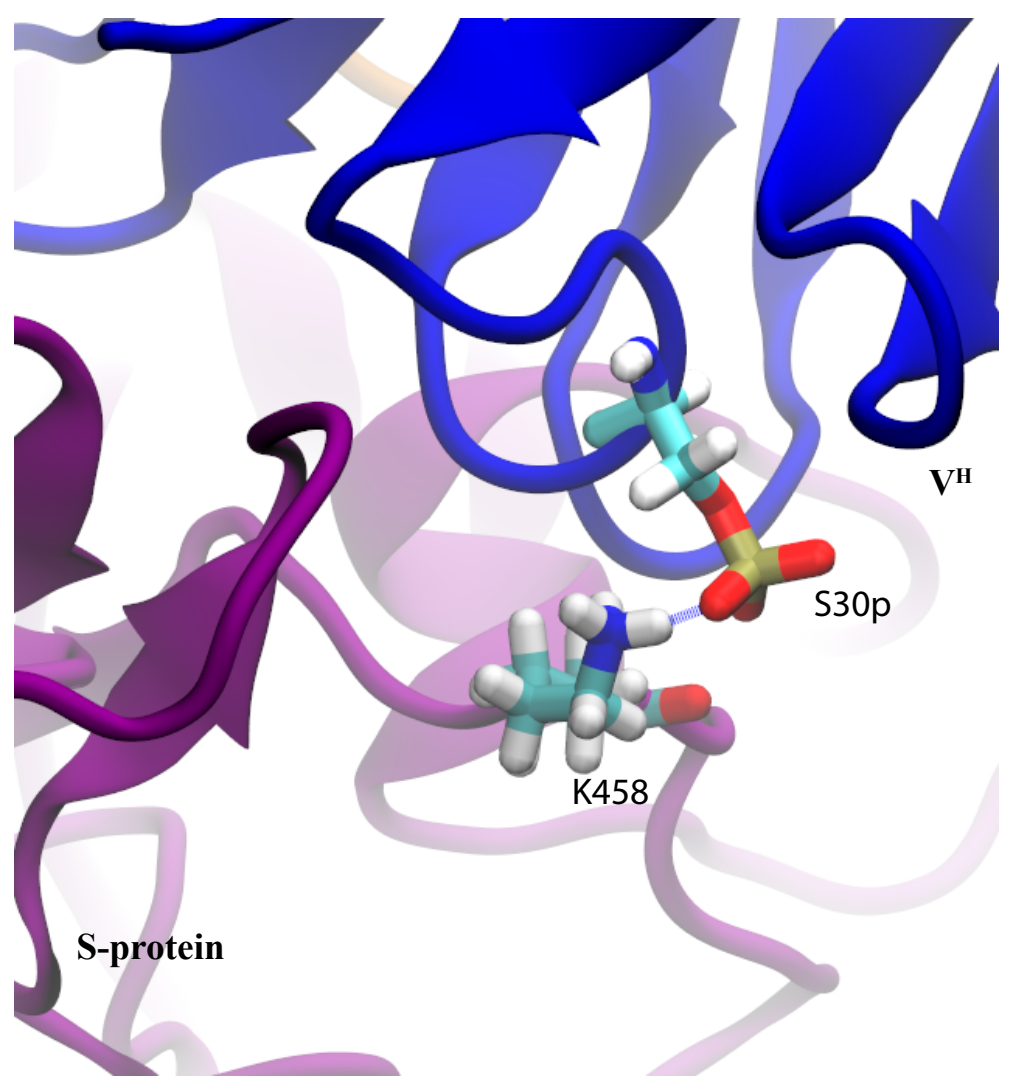

Figure S5: Illustration of local coordination between phosphorylated S30 (S30p) and K458 in the RBD. The salt-bridge between S30p and K458 was formed during the modification of S30 to its phosphorylated one (S30p). 

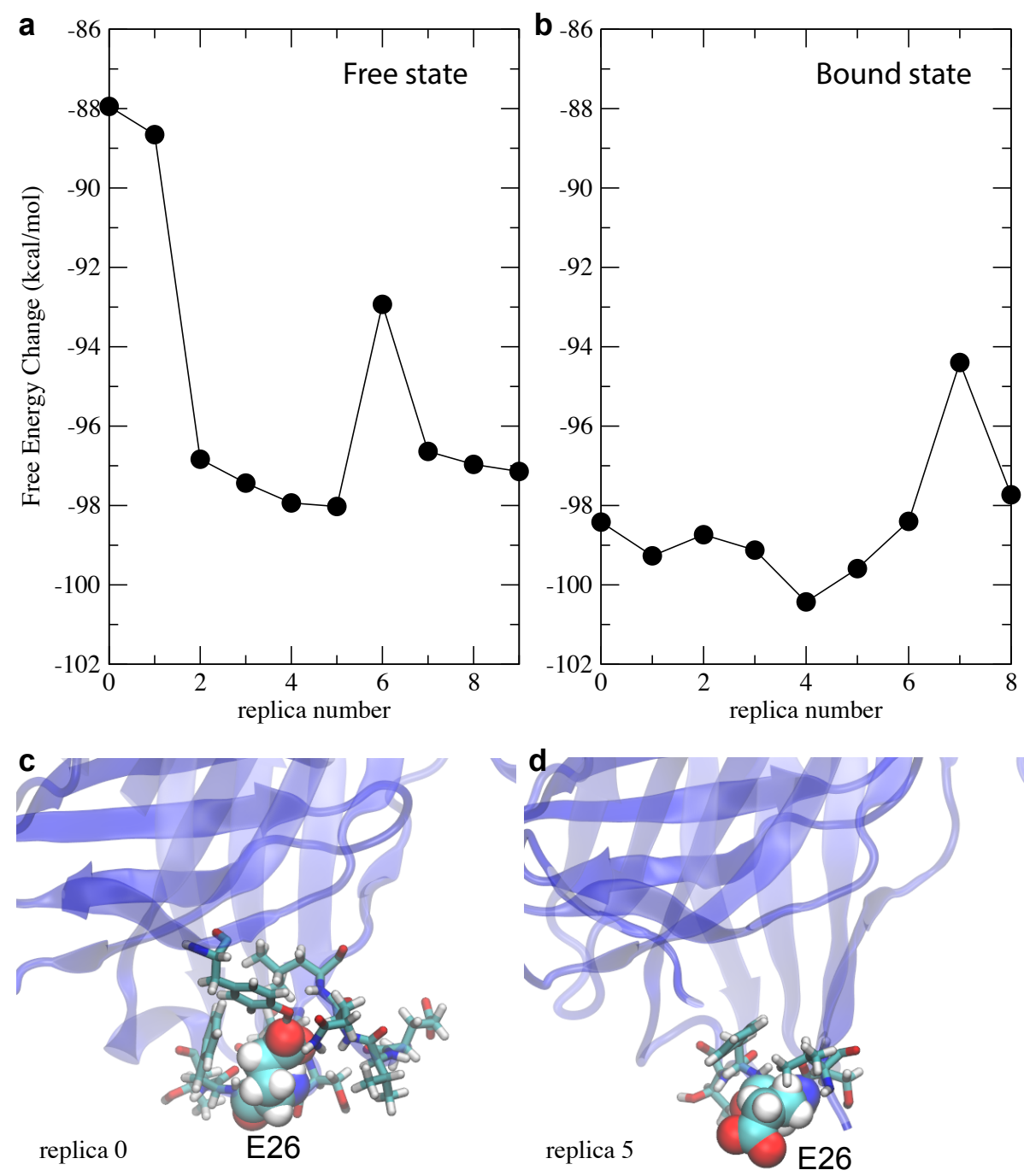

Figure S6: Free energy changes of the mutation G26E from different FEP runs. a) FEP runs in the free state. The average of the lowest five $\Delta G_{B}$ values is $-97.50 \mathrm{kcal} / \mathrm{mol}$. b) FEP runs in the bound state. The average of the lowest five $\Delta G_{B}$ values is $-99.43 \mathrm{kcal} / \mathrm{mol}$. Overall, $\Delta \Delta G=-1.93 \pm 0.32 \mathrm{kcal} / \mathrm{mol}$. c) Unfavorable conformation (buried) of the charged E26 after the FEP run in the replica 0 in the free state. $\Delta G_{B}=-87.95 \mathrm{kcal} / \mathrm{mol}$ (Fig. S6a), which was excluded from our data analysis. d) Favorable conformation (exposed to water) of the charged E26 after the FEP run in the replica 5 in the free state. $\Delta G_{B}=-98.02 \mathrm{kcal} / \mathrm{mol}$ (Fig. S6a). 


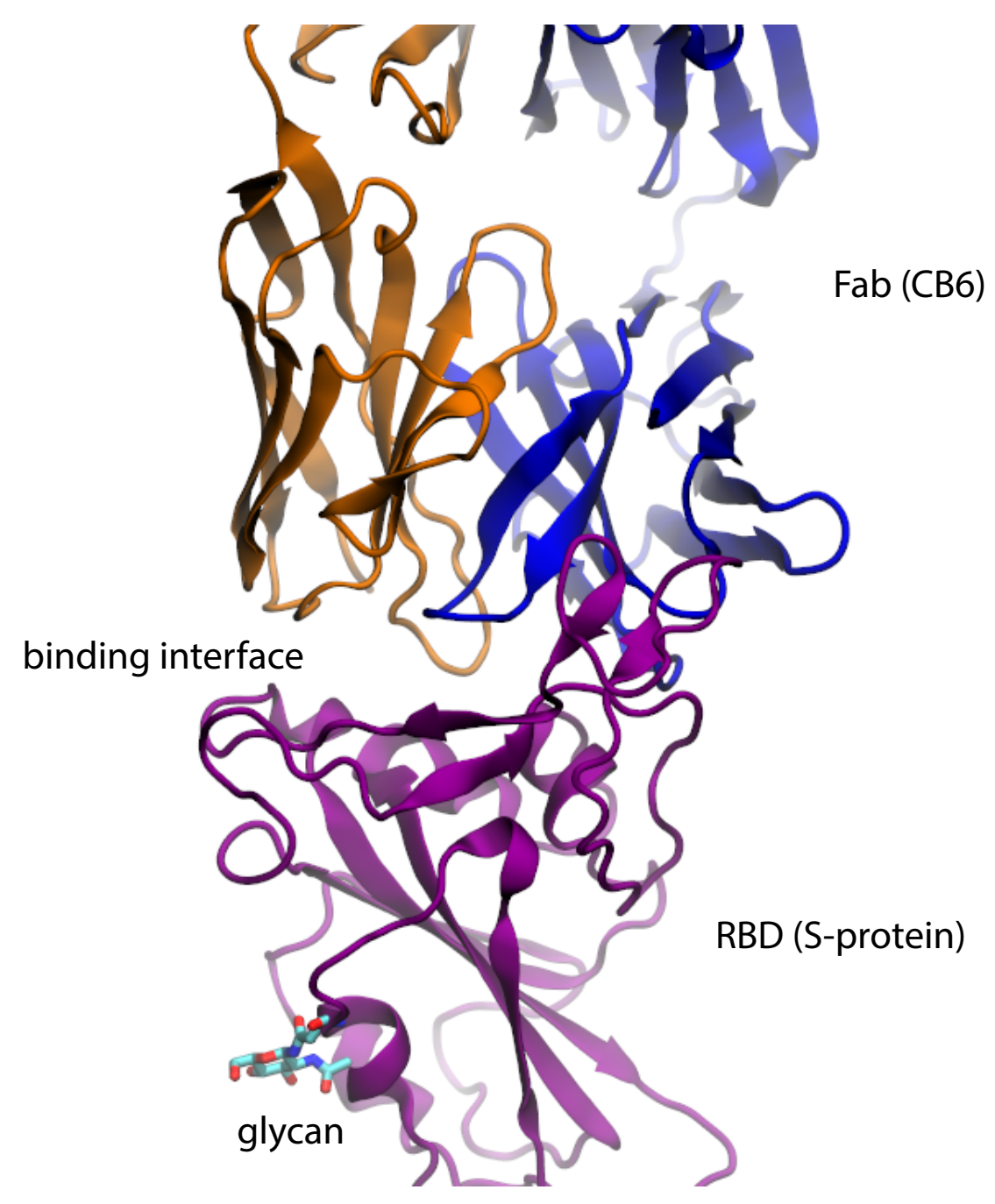

Figure S7: Illustration of a glycan molecule on RBD of S-protein. Note that the glycan link to the residue N343 of RBD is far away from the binding interface between CB6 and RBD. All mutations listed in Figs. 2 and 3 (in main paper) occurred at the binding interface. For the purpose of optimizing the binding affinity of CB6 to RBD, we did not include the glycan in this work. 